\title{
The Natural Antioxidants, Pomegranate Extract and Soy Isoflavones, Favourably Modulate Canine Endothelial Cell Function
}

\author{
Sabina M. Baumgartner-Parzer, ${ }^{1}$ Ferdinand Rudolf Waldenberger, ${ }^{2}$ \\ Angelika Freudenthaler, ${ }^{1}$ Amandine Ginouvès-Guerdoux, ${ }^{3}$ David McGahie,, 4 \\ and Hugues Gatto ${ }^{3}$ \\ ${ }^{1}$ Clinical Division of Endocrinology and Metabolism, Department of Internal Medicine III, \\ Medical University of Vienna, 1090 Vienna, Austria \\ ${ }^{2}$ Heart Center Hietzing, Wolkersbergstraße 1, 1130 Vienna, Austria \\ ${ }^{3}$ Unlicensed Product Development Unit, Virbac, 13ème rue, 06511 Carros Cedex, France \\ ${ }^{4}$ Medical Department, Virbac, 13 ème rue, 06511 Carros Cedex, France \\ Correspondence should be addressed to David McGahie, david.mcgahie@virbac.com
}

Received 8 October 2012; Accepted 26 October 2012

Academic Editors: Z. Grabarevic and W. Yang

Copyright () 2012 Sabina M. Baumgartner-Parzer et al. This is an open access article distributed under the Creative Commons Attribution License, which permits unrestricted use, distribution, and reproduction in any medium, provided the original work is properly cited.

Cardiovascular disease, preceded by vascular endothelial dysfunction, is a prominent cause of death in dogs. L-carnitine and taurine, well known for their antioxidative capacity, beneficially affect cardiovascular disease as well as certain dog cardiomyopathies. It is well established that vascular endothelial dysfunction precedes cardiovascular disease and that "vasoprotective factors" (NO and antioxidants) prevent apoptosis, whereas "risk factors" such as oxidized LDL, hyperglycemia, and free fatty acids trigger it in cultured human vascular endothelial cells. Whereas human vascular cell in vitro models are widely established and used for the characterisation of potential vasoprotective substances, such models are not available for canine endothelial cells. In the present study we therefore developed an in vitro model, which allows the testing of the effects of different substances on proliferation and apoptosis in canine aortic endothelial cells. This model was used to test L-carnitine, taurine, pomegranate extract, and Soy Isoflavones in comparison to reference substances (glutathione and pioglitazone) previously shown to modulate human endothelial cell function. L-carnitine and taurine neither exhibited antiproliferative nor antiapoptotic activities in the context of this study. However extracts from pomegranate and soy isoflavones dramatically reduced proliferation and apoptosis in a dose dependent fashion, being in line with a vasoprotective activity in dogs.

\section{Introduction}

The increased life expectancy of dogs over recent years, probably due mainly to advances in canine nutrition and health care, has also been associated with an increased prevalence of cardiovascular disease [1]. The vascular endothelium represents a widespread and interactive organ with various biological actions including barrier function, secretion of anti/prothrombotic factors, leukocyte and platelet adhesion and, very importantly, regulation of vascular tone $[2,3]$. Macrovascular disease and heart failure are preceded and predicted by increased apoptosis of endothelial cells and dysfunction of the vascular endothelium including increased endothelial cell turnover to maintain an intact endothelial lining, increased smooth muscle cell migration due to an impaired endothelial barrier function, and a loss of vascular elasticity leading to an increased afterload [2, 46]. Moreover, it is well established that heart failure, experimentally induced in dogs by rapid pacing [7], as well as vascular endothelial dysfunction relate to reduced generation and/or exaggerated degradation of nitric oxide (NO). Reduced bioavailability of $\mathrm{NO}$ is associated with exaggerated 
superoxide anion production, increased oxidative stress, and sustained vasoconstriction $[2,8]$. Such effects are believed to trigger vascular endothelial cell apoptosis and, in the long run, result in progression of human and canine valvular disease and heart failure [9-12]. Therefore, identification and characterization of vasoprotective agents and of their effects on endothelial cell function are of major importance in order to prevent or ameliorate the sequelae of endothelial dysfunction and vascular disease.

So far L-carnitine and taurine, both known for their antioxidative capacity $[13,14]$, have been shown to be associated with beneficial effects in human endothelial cells as well in certain dog cardiomyopathies [15-22]. Similarly, polyphenolic compounds such as isoflavones found in soybeans and tannins present in pomegranate extracts exhibit antioxidant and cyto- and cardioprotective activities [2330]. Concerning the human vascular endothelium, it has been proven by a considerable number of studies that factors often termed "vasoprotective factors" (NO, shear stress, antioxidative agents) prevent, whereas "atherosclerotic risk factors" (oxidized/glycated LDL, hyperglycemia, proinflammatory cytokines, elevated free fatty acids) trigger apoptosis in cultured human vascular endothelial cells [31-39].

Due to a lack of such models for the canine vascular endothelium, it was largely unknown to which extent the beneficial effects of L-carnitine, taurine, soy isoflavones, and pomegranate extract for the cardiovascular system relate to direct effects of those antioxidants on canine endothelial cell health and function. A previous recent study demonstrated that they could decrease the loss of viability of canine aortic endothelial cells (CnAoEC) under conditions of oxidative stress [40]. However the viability assay used in that study relied on total metabolic activity, and therefore it could be theorised that there is a risk of artificially good results if the tested substances concurrently promote significant excessive proliferation in surviving cells. Information is therefore still lacking regarding the impact of these substances on key indicators of functional health in canine endothelial cells such as apoptosis and proliferation. Moreover, it remains to be elucidated whether the observed effects of substances known to modulate these parameters are species dependent or can be extrapolated from species to species [41].

Therefore, the present study aimed at development of an in vitro model on the basis of CnAoEC, in order to characterize the potential beneficial or detrimental effects of different test agents with respect to canine endothelial cell proliferation and apoptosis. Different substances (GSH, NAc, insulin sensitizers, etc.), previously well characterized in human in vitro models for endothelial dysfunction [3438 ], were used as putative references for the development of our canine model and as internal controls for subsequent assays performed with test agents. We hypothesised that the reference substances would produce similar effects in CnAoEC to those seen with human endothelial cells. We further hypothesised that the previously noted beneficial effect of the four test substances [40] would be associated with stable or decreased apoptosis and proliferation, thus confirming the interest of these substances in developing a multidimensional dietary strategy to reduce the onset and progression of the canine endothelial degeneration involved in progressive valvular diseases.

\section{Research Design and Methods}

2.1. Reagent Sources. CnAoECs and the respective media (CECBM, HBSS) and growth supplements were purchased from Cell Applications, Inc. (San Diego, USA). Linoleic acid (LoIS), $\gamma$-linolenic acid (ALens), fibronectin, bovine Serum albumin (BSA), dimethyl sulfoxide (DMSO), glutathione (GSH), and N-acetylcysteine (NAc) were purchased from Sigma Chemical Co. Phosphate buffered saline (PBS) and trypsin ethylenediaminetetraacetic acid (trypsin EDTA) were from BioWhittaker/Lonza (Belgium), human vascular endothelial growth factor (VEGF) and basic fibroblast growth factor (bFGF) were purchased from BioVision, and [Methyl- ${ }^{3} \mathrm{H}$ ] thymidine from Amersham Pharmacia. Taurine and L-carnitine L-tartrate were from Azelis Pharma (Paris, France), pomegranate extract (40\% punicosides) from Polinat (Las Palmas, Spain), and soy extract (standardized at $40 \%$ soy isoflavones) from ADM (Decatur, IL, USA).

\subsection{Test and Reference Substances}

2.2.1. Reference Substances. GSH ( $10 \mathrm{mM}$, equivalent to $3.1 \mathrm{mg} / \mathrm{mL}$ ), NAc ( $5 \mathrm{mM}$, equivalent to $816 \mu \mathrm{g} / \mathrm{mL}$ ), VEGF (25 ng $/ \mathrm{mL})$, bFGF (10 ng /mL), LoIS $(50 \mu \mathrm{M}$, equivalent to $14 \mu \mathrm{g} / \mathrm{mL})$, ALenS $(50 \mu \mathrm{M}$, equivalent to $13.9 \mu \mathrm{g} / \mathrm{mL})$, and the insulin sensitizers Pioglitazone (Pio) ( $50 \mu \mathrm{M}$, equivalent to $17.8 \mu \mathrm{g} / \mathrm{mL}$ ) and Rosiglitazone (Rosi) ( $50 \mu \mathrm{M}$, equivalent to $17.9 \mu \mathrm{g} / \mathrm{mL}$ ) were prepared and used as previously described [34-38]. In brief, free fatty acids (LoIS and ALenS) were dissolved in ethanol, the insulin sensitizers (Pio and Rosi) were dissolved in DMSO, all other reference substances were soluble in water. Where DMSO or ethanol were required for the test or reference substances, the respective control cultures had the equivalent concentrations of DMSO (1\%) or ethanol $(1.5 \%)$, respectively.

2.2.2. Test Substances. L-carnitine, taurine, pomegranate extract, and soy extract were kept dry and protected from light (in brown flasks, covered with parafilm (bottle tops) and with aluminium foil). Before each experiment, fresh stock solutions were prepared for each substance: soy isoflavones were dissolved in DMSO $(250 \mathrm{mg} / \mathrm{mL})$, all other test substances were soluble in water $(50 \mathrm{mg} / \mathrm{mL})$. Where DMSO was required for the test substances, the respective control cultures had the equivalent concentrations of $1 \%$ DMSO. The sample concentrations to be tested in the different assays, that is, 1,50 , and $250 \mu \mathrm{g} / \mathrm{mL}$, were estimated by reference to existing published studies using human endothelial cells or data reporting plasma concentrations in dogs or humans following oral supplementation $[15,19,42-$ 44 ], and the absence of cytotoxicity of the proposed levels of these substances on CnAoEC (data not shown).

Experimental wells (with test or reference substances) were related to the respective control wells (without test or reference substances), set to $100 \%$. 


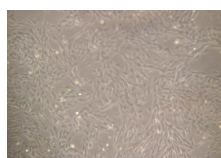

CnAoEC 96 well plate (6 hrs to adhere)

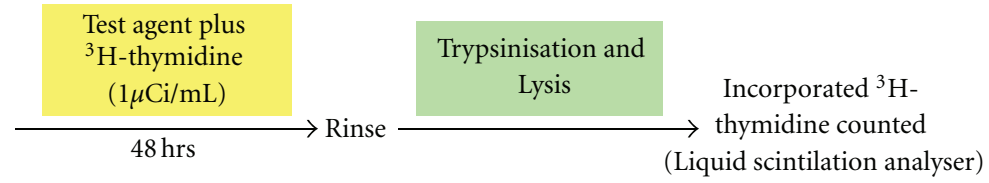

(Liquid scintilation analyser)

Figure 1: Proliferation Assay.

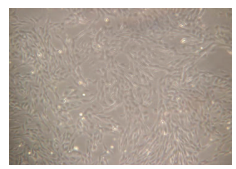

CnAoEC Semiconfluent in $60 \mathrm{~mm}$ plates

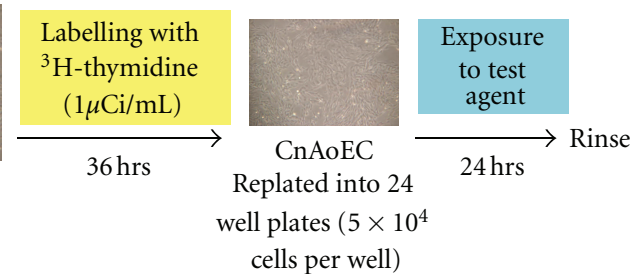

cells per well)



Figure 2: Apoptosis Assay.

2.3. Canine Aortic Endothelial Cell Culture. CnAoECs received as cryopreserved vial containing 500000 cells were immediately frozen in liquid nitrogen and kept there until use. For further use, cells were thawed at $37^{\circ} \mathrm{C}$, resuspended in CECGM (according to the manufacturer's instructions), and cultured on Fibronectin $(0.0025 \%$ in PBS) coated $\left(2 \mathrm{~h} / 37^{\circ} \mathrm{C}\right)$ cell culture dishes at $37^{\circ} \mathrm{C} / 5 \% \mathrm{CO}_{2}$.

For subcultures, confluent cells were rinsed with HBSS and treated with trypsin/EDTA $\left(6 \mathrm{~mL} / 75 \mathrm{~cm}^{2}\right)$, followed by addition of $8 \mathrm{~mL}$ trypsin neutralisation solution (5\% BSA in PBS). After centrifugation $(5 \mathrm{~min} / 220 \mathrm{~g}$ ) the collected cells were replated in CECGM with a density of 5000 to 10000 cells $/ \mathrm{cm}^{2}$.

The day after seeding and every other day, the medium was changed and confluency of the cells monitored by phase contrast microscopy.

For proliferation and apoptosis assays, cells were used between passages 5 and 7 .

\subsection{Proliferation Assays. Proliferation assays (see Figure 1)} were carried out as described previously [34, 35, 37]. In brief, confluent CnAoECs cultures were replated in flat bottomed 96-well tissue culture plates (10,000 cells per well) and allowed to adhere for $6 \mathrm{~h}$. Subsequently, cells were exposed to ${ }^{3} \mathrm{H}$-thymidine (final concentration: $1 \mu \mathrm{Ci} / \mathrm{mL}=$ $37 \mathrm{kBq} / \mathrm{mL}$ ) and the respective test agents for $48 \mathrm{~h}$. After two washing steps with PBS, cells were trypsinized, lysed by a freeze thaw cycle, harvested, and incorporated. ${ }^{3} \mathrm{H}$ thymidine was counted in a Tri-Carb Liquid Scintillation Analyser (Canberra Packard, Meriden, USA). Samples were tested in quadruplicates. Results of experimental cultures exposed to the test substances are presented in relation to intraindividual control cultures (without test substances). Control cultures were set to $100 \%$.
2.5. Apoptosis Assays. Apoptosis assays (see Figure 2) were performed as previously described [34, 35, 39]. In brief, semiconfluent plates $(60 \mathrm{~mm})$ of CnAoECs were labelled with ${ }^{3} \mathrm{H}$-thymidine $(37 \mathrm{kBq} / \mathrm{mL}, 36 \mathrm{~h})$ and were subsequently replated into 24 -well culture plates $(5 \times 104$ cells/well $)$. After their exposure to the test substances ( 24 hours) cells were treated with lysis buffer $(20 \mathrm{mmol} / \mathrm{l}$ Tris.Cl, $\mathrm{pH} 7.5$, and $0.4 \%$ Triton X-100 in PBS). Fragmented (apoptotic) radiolabeled DNA in the supernatant was counted in a Liquid Scintillation Analyser (Canberra Packard, Meriden, CT) and was then related to total incorporated radioactivity of cells (quantified after digestion of the remaining suspension with $180 \mu \mathrm{g} / \mathrm{mL}$ DNase, Boehringer Mannheim, Germany). Experiments were performed in triplicates or duplicates. Results of experimental cultures exposed to test substances are presented in relation to intraindividual control cultures (without test substances). Control cultures were set to $100 \%$.

2.6. Statistics. Data are expressed as means \pm SD. Statistical analysis was performed using Student's paired $t$-test.

\section{Results}

\subsection{Proliferation}

3.1.1. Reference Substances. As depicted in Figure 3, the antioxidant GSH, the insulin sensitizers Pio and Rosi, and the free fatty acids LoIS and ALenS significantly reduced proliferation in CnAoECs, such results being in line with previous work in different types of human vascular endothelial cells $[35,37]$. Of note, however, VEGF and bFGF, known to increase proliferation in human endothelial cells [34], did not provoke such proproliferative response in canine cells. 


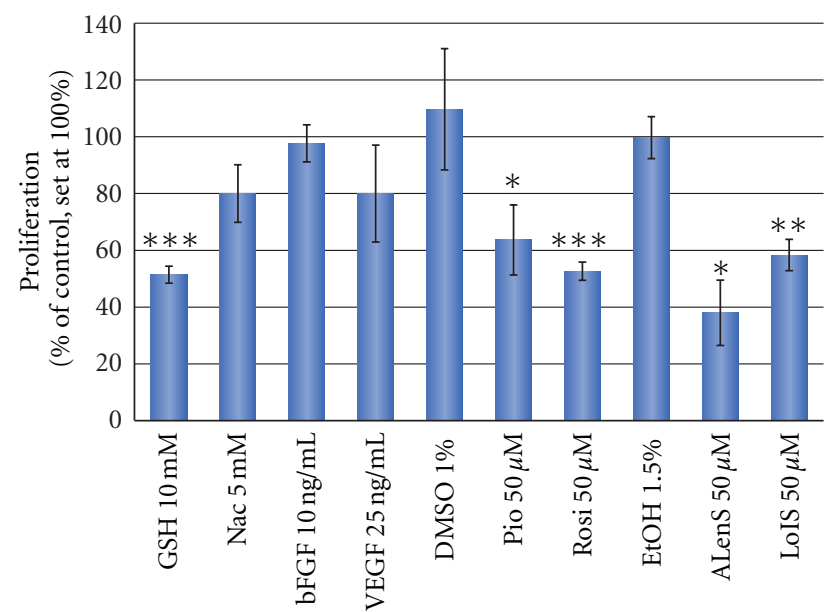

FIgURE 3: Impact of selected reference substances on the proliferation of CnAoECs (mean of 3 independent experiments each performed in 4 wells) ${ }^{*} P<0.05 ;{ }^{* *} P<0.01 ;{ }^{* * *} P<0.005$.

Ethanol and DMSO, used as solvents for FFAs and insulin sensitizers, respectively, did not significantly affect CnAoECs proliferation.

3.1.2. Test Substances. In CnAoECs, L-carnitine and taurine induced a slight but significant reduction of proliferation by $6.25 \%$ and $10.25 \%$, respectively, at the highest concentration only $(250 \mu \mathrm{g} / \mathrm{mL}$, see Figure $4(\mathrm{a}))$. However, pomegranateextract and soy isoflavones markedly reduced CnAoECs proliferation, even at a concentration of $50 \mu \mathrm{g} / \mathrm{mL}$, by $90 \%$ and 25\%, respectively, and in a dose-dependent manner (Figure 4(b)). Exposure of CnAoECs to $250 \mu \mathrm{g} / \mathrm{mL}$ of these two substances led to an impressive and highly significant inhibition of the cells' proliferation to $3.75 \%$ and $15.5 \%$ of control (set to $100 \%$ ), whereas Pio and GSH reduced proliferation only to $62.25 \%$ and $54.75 \%$ of control, respectively (Figure 4(b)).

\subsection{Apoptosis}

3.2.1. Reference Substances. As shown in Figure 5(a), the antioxidant GSH at $10 \mathrm{mM}$ markedly reduced apoptosis (by 35\%) in CnAoECs. In contrast, in CnAoECs exposed to $300 \mu \mathrm{mol} / \mathrm{L}$ LoIS and ALenS, death rates dramatically increased, more than $55 \%$ of cells having undergone apoptosis after $48 \mathrm{~h}$ of incubation (data not shown). Such antiapoptotic effects of antioxidants as well as the proapoptotic response exerted by free fatty acids have previously been observed in human vascular endothelial cells $[34,35]$.

3.2.2. Test Substances. L-carnitine and taurine did not markedly affect apoptosis in CnAoECs, the statistical significance observed for $50 \mu \mathrm{g} / \mathrm{mL}$ Carnitine being probably related to the very low standard deviation observed for that particular concentration (Figure 5(a)).

The antiapoptotic effect of soy extract was significant only for the highest concentration tested $(250 \mu \mathrm{g} / \mathrm{mL})$.
In contrast, pomegranate extracts significantly reduced apoptosis at all concentrations tested and in a dose dependent fashion, showing the most striking effect at $250 \mu \mathrm{g} / \mathrm{mL}$ by a reduction of $73 \%$ compared to control cells (Figure $5(b))$.

\section{Discussion}

Due to the increasing amount of evidence linking endothelial dysfunction to the onset and progression of heart failure in dogs $[11,12]$, it is important to have a method of screening and/or testing substances that may have potential to protect endothelial function in vitro. This is already widely done with human endothelial cells, and the markers of excessive proliferation and apoptosis are crucial indicators of dysfunction. However, until now, no such model existed using canine endothelial cells. This is why we began the study with the establishment of an in vitro cell culture model which allowed the reproducible analysis of these markers in response to different stimuli. This then enabled, as a second step, the identification of pro- and antiproliferative as well as of pro- and antiapoptotic agents.

The reference substances were chosen on the basis of their abilities to exert anti- as well as proproliferative and anti- and proapoptotic responses in human endothelial cell culture models. Of note, the observed antiapoptotic and antiproliferative effects were similar in human endothelial cells and the tested canine endothelial cells for the antioxidants GSH and NAc as well as for the insulin sensitizers Pio and Rosi $[34,35,37]$. The free fatty acids exhibit marked proapoptotic action in canine endothelial cells, which clearly exceeds the effects exerted in human vascular endothelial cells [35]. Of course it must be noted that this relates to use of these particular free fatty acids in isolation, as opposed to the more normal dietary situation where a balance of Omega-3 and Omega- 6 oils will be present, and where the ratio of these oils has already been shown to be important [45]. In contrast to human umbilical vein endothelial cells [34], VEGF and bFGF, were not able to provoke a proproliferative response in CnAoECs. In that context it is of note that it has previously been speculated that the effects of natural antioxidant molecules in endothelial cells can be species dependent [41]. As could be observed for the reference substances GSH and PIO, which were used as internal controls in each assay performed for the test substances, the model exhibited considerable reproducibility throughout the whole study, even if different settings (coating, growth factor supplement, etc.) were employed (data not shown). This therefore partially confirms our initial hypothesis that substances shown to be beneficial for human endothelial cells will also produce beneficial results in CnAoECs, but intriguingly suggests that it is not possible to extrapolate these results directly across species due to occasional differences in the intensity of the effects. This confirms the need to test substances in cells derived from the target species.

For the first time this study shows that extracts of pomegranate and soy isoflavones are able to exhibit dramatic antiapoptotic activities in CnAOECs. Pomegranate extract's 


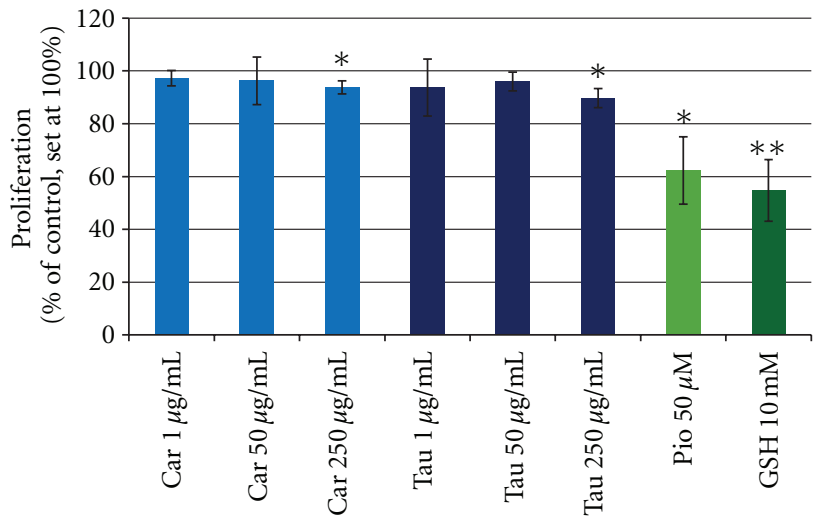

(a)

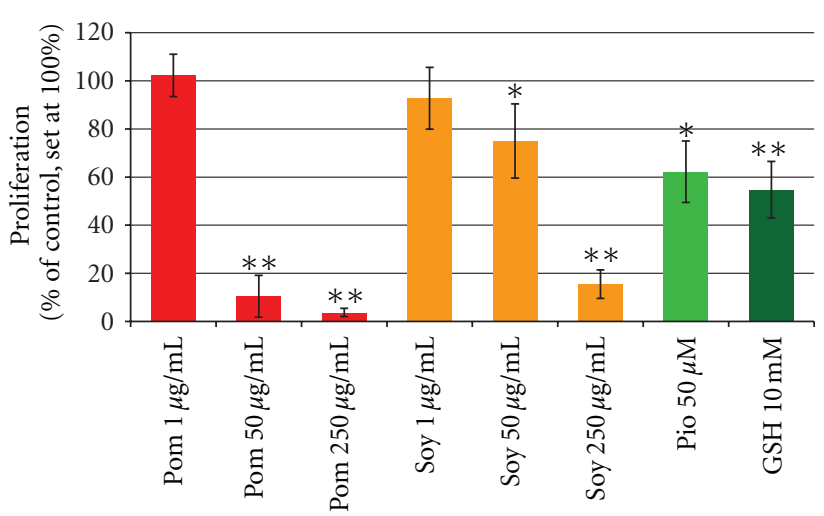

(b)

FIgURE 4: (a) Modulation of proliferation of CnAoECs by carnitine and taurine in comparison to the reference substances Pio and GSH (mean of 4 experiments, each in 4 wells) ${ }^{*} P<0.05 ;{ }^{*} P<0.01$. (b) Modulation of proliferation of CnAoECs by pomegranate extract and soy isoflavone extract in comparison to the reference substances Pio and GSH (mean of 4 experiments, each in 4 wells) ${ }^{*} P<0.05 ; * * P<0.01$.

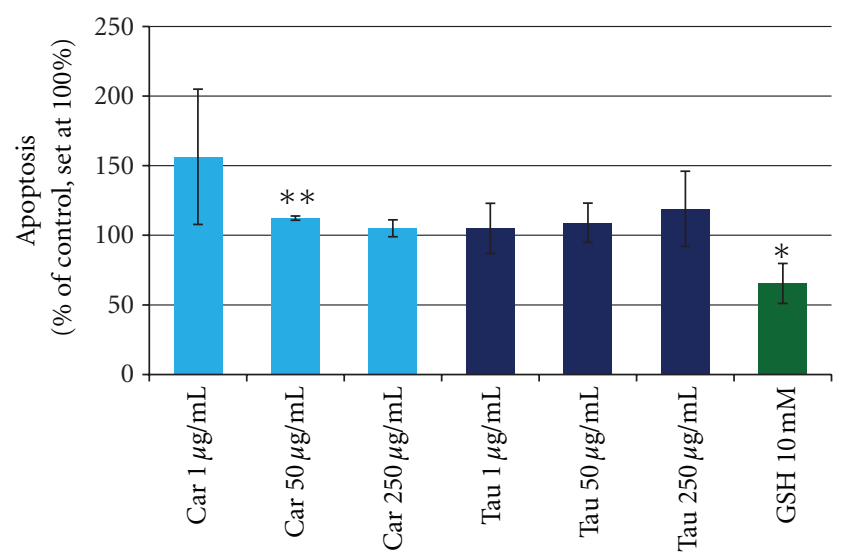

(a)

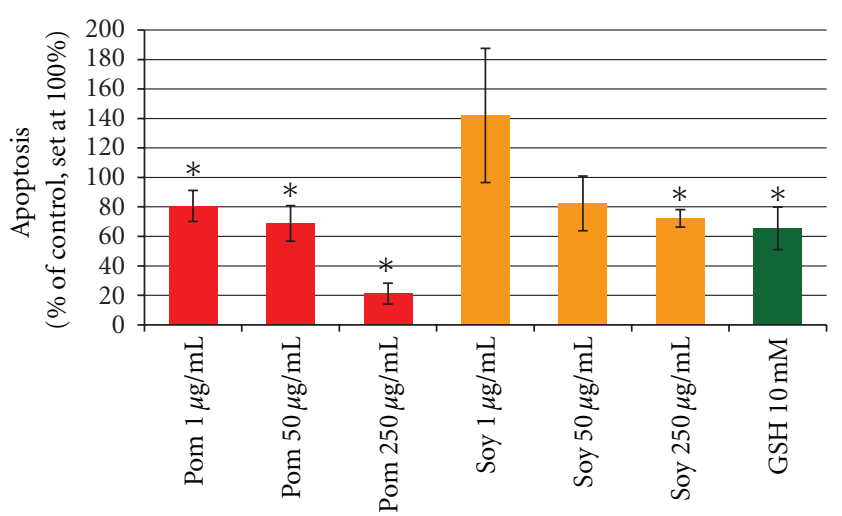

(b)

FIGURE 5: (a) Modulation of apoptosis in CnAoECs by carnitine and taurine in comparison to the reference substance GSH (means of 3 independent experiments, each in 3 wells) $* P<0.05$; $* *<0.01$. (b) Modulation of apoptosis in CnAoECs by pomegranate extract and soy isoflavone extract in comparison to the reference substance GSH (means of 3 independent experiments, each in 3 wells) ${ }^{*} P<0.05$.

antiapoptotic effect was already detectable at the lowest concentration tested $(1 \mu \mathrm{g} / \mathrm{mL})$ and was dose-dependently sustained until the highest concentration applied $(250 \mu \mathrm{g} / \mathrm{mL})$. Accelerated apoptosis of vascular endothelial cells is relevant in the development and progression of cardiovascular disease [4-6]. We have previously shown that vasoprotective agents such as antioxidants (including lipoic acid and GSH) or insulin sensitizers exhibit both antiapoptotic and antiproliferative effects in human vascular endothelial cells. Those effects could prevent loss of the endothelial barrier function (due to accelerated endothelial cell apoptosis) and exhaustion of the endothelial cells' proliferative capacity (due to accelerated proliferation). Both the antiapoptotic and antiproliferative activity of pomegranate and isoflavones in CnAoECs therefore suggest a vasoprotective action of those polyphenolic compounds in dogs, which could beneficially affect chronic mitral valvular insufficiency (CMVI), the major cause of heart failure in this species [1]. Since progression of CMVI is assumed to be triggered by endothelial dysfunction and the latter vice versa is potentiated by CMVI-associated increased cellular oxygen demand $[4,11$, $12,17,46-48]$ the cytoprotective antiapoptotic as well as antioxidative effects of pomegranate [49] and soy isoflavone extracts could beneficially affect the progression of CMVI to heart failure in dogs.

Both of these agents are complex biological substances containing multiple potentially active polyphenols or isoflavones. It was not possible or appropriate to attempt to identify the balance of the effects of individual components of complex natural substances such as these, but indeed this kind of complexity using agents with a multi-faceted action has been proposed as a necessary and important part of rational use of antioxidants to prevent cardiovascular disease [50].

L-carnitine and taurine were previously shown to exert protective effects against oxidative stress in human endothelial cells $[15,22]$. In a recent study we have shown that pomegranate extract, alone and in combination with soy 
isoflavones, taurine, and L-carnitine, also shows strong protective effects against oxidative stress in CnAoECs [40]. In that context it is of note that the survival signaling pathways of the Bcl-2 family, PI3Kinase, and estrogen receptor are assumed to mediate the protective antioxidative effects of the soy-derived isoflavones genistein and daidzein [30]. Such pathways have previously been shown to mediate antiapoptotic effects described for lipoic acid [34] in human vascular endothelial cells. Since taurine and L-carnitine, in contrast to extracts of pomegranate and soy isoflavones, did not affect apoptosis in canine aortic endothelial cells, it is tempting to speculate that different mechanisms are involved in those substances' antioxidative and beneficial vascular effects in dogs. It is also worth noting that any results obtained for extracts of pomegranate are probably specific to each type of extract. It has been shown that extracts obtained from different parts of the plant have widely varying compositions of active components $[49,51]$.

Concerning the concentrations applied, there is a wide range of concentrations described in the literature for antioxidants, depending on species and compound. To cover such variety the test substances were used in a broad range from 1 to $250 \mu \mathrm{g} / \mathrm{mL}$. The lack of any cytotoxic effects at the highest concentrations we used also provides some reassurance regarding the potential use of these compounds.

One limitation of a study such as this is related to the use of an in vitro cell-based model. Indeed it is possible that there may be differences in the responses of cells in the in vivo situation compared to the in vitro environment, and it is also not possible to assess completely the impact of the diseased state on the function of the endothelial cells. One possible solution could be to use isolated segments of blood vessel to assess vasorelaxation responses. However this equally would have significant limitations and would preclude the direct assessment of key markers such as apoptosis. On balance, as substances having beneficial effects on proliferation and apoptosis of human endothelial cells in the in vitro situation have been shown to also provide in vivo benefits, this type of study is a rational step providing key information regarding the potential benefits of these substances in the canine species.

In conclusion, this is the first study showing that pomegranate extract and soy isoflavones inhibit apoptosis and proliferation in canine aortic endothelial cells. This supports our hypothesis that these agents could have a role in prevention or amelioration of clinical endothelial dysfunction and therefore progression of cardiovascular disease in dogs $[47,48]$. Further studies will, however, be necessary to evaluate the underlying mechanisms and to which extent the antiapoptotic and antiproliferative effects observed in our canine endothelial cell culture model could also apply to the in vivo situation.

\section{Abbreviations}

bFGF: Basic fibroblast growth factor

CnAoECs: Canine aortic endothelial cells

CECBM: Canine endothelial cell basal medium

CECGM: Canine endothelial cell growth medium
FFA: Free fatty acid

FN: Fibronectin

GSH: Glutathione

HBSS: Hank's Buffered Salt Solution

ALenS: $y$-Linolenic acid

LolS: Linoleic acid

NAc: N-Acetylcysteine

Pio: Pioglitazone

Rosi: Rosiglitazone

SD: $\quad$ Standard deviation

VEGF: Vascular endothelial growth factor.

\section{Conflict of Interests}

Authors A. Ginouvès-Guerdoux, D. McGahie, and H. Gatto are employees of Virbac. Virbac paid for this research which relates to a potential future product in development.

\section{References}

[1] C. Guglielmini, "Cardiovascular diseases in the ageing dog: diagnostic and therapeutic problems," Veterinary Research Communications, vol. 27, supplement 1, pp. 555-560, 2003.

[2] R. Belardinelli, "Endothelial dysfunction in chronic heart failure: clinical implications and therapeutic options," International Journal of Cardiology, vol. 81, no. 1, pp. 1-8, 2001.

[3] S. M. Baumgartner-Parzer and W. K. Waldhäusl, "The endothelium as a metabolic and endocrine organ: Its relation with insulin resistance," Experimental and Clinical Endocrinology and Diabetes, vol. 109, no. 2, pp. S166-S179, 2001.

[4] T. Scarabelli, A. Stephanou, N. Rayment et al., "Apoptosis of endothelial cells precedes myocyte cell apoptosis in ischemia/reperfusion injury," Circulation, vol. 104, no. 3, pp. 253256, 2001.

[5] L. Rössig, S. Dimmeler, and A. M. Zeiher, "Apoptosis in the vascular wall and atherosclerosis," Basic Research in Cardiology, vol. 96, no. 1, pp. 11-22, 2001.

[6] J. C. Choy, D. J. Granville, D. W. C. Hunt, and B. M. McManus, "Endothelial cell apoptosis: Biochemical characteristics and potential implications for atherosclerosis," Journal of Molecular and Cellular Cardiology, vol. 33, no. 9, pp. 1673-1690, 2001.

[7] A. Leri, Y. Liu, A. Malhotra et al., "Pacing-induced heart failure in dogs enhances the expression of p53 and p53-dependent genes in ventricular myocytes," Circulation, vol. 97, no. 2, pp. 194-203, 1998.

[8] P. Korantzopoulos, D. Galaris, D. Papaioannides, and K. Siogas, "The possible role of oxidative stress in heart failure and the potential of antioxidant intervention," Medical Science Monitor, vol. 9, no. 6, pp. RA120-RA125, 2003.

[9] M. L. Rossi, N. Marziliano, P. A. Merlini et al., "Different quantitative apoptotic traits in coronary atherosclerotic plaques from patients with stable angina pectoris and acute coronary syndromes," Circulation, vol. 110, no. 13, pp. 1767-1773, 2004.

[10] Q. M. Chen and V. C. Tu, "Apoptosis and heart failure: Mechanisms and therapeutic implications," American Journal of Cardiovascular Drugs, vol. 2, no. 1, pp. 43-57, 2002.

[11] S. G. Moesgaard, C. Klostergaard, N. E. Zois et al., "Flowmediated vasodilation measurements in cavalier king charles spaniels with increasing severity of myxomatous mitral valve disease," Journal of Veterinary Internal Medicine, vol. 26, pp. 61-68, 2012. 
[12] S. M. Cunningham, J. E. Rush, and L. M. Freeman, "Systemic inflammation and endothelial dysfunction in dogs with congestive heart failure," Journal of Veterinary Internal Medicine, vol. 26, pp. 547-557, 2012.

[13] O. I. Aruoma, B. Halliwell, B. M. Hoey, and J. Butler, "The antioxidant action of taurine, hypotaurine and their metabolic precursors," Biochemical Journal, vol. 256, no. 1, pp. 251-255, 1988.

[14] I. Gülçin, "Antioxidant and antiradical activities of L-carnitine," Life Sciences, vol. 78, no. 8, pp. 803-811, 2006.

[15] L. A. Calò, E. Pagnin, P. A. Davis et al., "Antioxidant effect of L-carnitine and its short chain esters: relevance for the protection from oxidative stress related cardiovascular damage," International Journal of Cardiology, vol. 107, no. 1, pp. 54-60, 2006.

[16] L. M. Freeman, J. E. Rush, A. K. Cahalane, P. M. Kaplan, and P. J. Markwell, "Evaluation of dietary patterns in dogs with cardiac disease," Journal of the American Veterinary Medical Association, vol. 223, no. 9, pp. 1301-1305, 2003.

[17] L. M. Freeman, J. E. Rush, P. E. Milbury, and J. B. Blumberg, "Antioxidant status and biomarkers of oxidative stress in dogs with congestive heart failure," Journal of Veterinary Internal Medicine, vol. 19, no. 4, pp. 537-541, 2005.

[18] J. D. Folts, A. L. Shug, J. R. Koke, and N. Bittar, "Protection of the ischemic dog myocardium with carnitine," American Journal of Cardiology, vol. 41, no. 7, pp. 1209-1214, 1978.

[19] M. D. Kittleson, B. Keene, P. D. Pion, and C. G. Loyer, "Results of the multicenter spaniel trial (MUST): taurineand carnitine-responsive dilated cardiomyopathy in American cocker spaniels with decreased plasma taurine concentration," Journal of Veterinary Internal Medicine, vol. 11, no. 4, pp. 204211, 1997.

[20] S. L. Sanderson, K. L. Gross, P. N. Ogburn et al., "Effects of dietary fat and L-carnitine on plasma and whole blood taurine concentrations and cardiac function in healthy dogs fed protein-restricted diets," American Journal of Veterinary Research, vol. 62, no. 10, pp. 1616-1623, 2001.

[21] S. L. Sanderson, "Taurine and carnitine in canine cardiomyopathy," Veterinary Clinics of North America-Small Animal Practice, vol. 36, no. 6, pp. 1325-1343, 2006.

[22] B. Tan, D. J. Jiang, H. Huang et al., "Taurine protects against low-density lipoprotein-induced endothelial dysfunction by the DDAH/ADMA pathway," Vascular Pharmacology, vol. 46, no. 5, pp. 338-345, 2007.

[23] C. Manach, A. Mazur, and A. Scalbert, "Polyphenols and prevention of cardiovascular diseases," Current Opinion in Lipidology, vol. 16, no. 1, pp. 77-84, 2005.

[24] S. K. Nicholson, G. A. Tucker, and J. M. Brameld, "Effects of dietary polyphenols on gene expression in human vascular endothelial cells," Proceedings of the Nutrition Society, vol. 67, no. 1, pp. 42-47, 2008.

[25] G. Rimbach, S. De Pascual-Teresa, B. A. Ewins et al., "Antioxidant and free radical scavenging activity of isoflavone metabolites," Xenobiotica, vol. 33, no. 9, pp. 913-925, 2003.

[26] J. Sierens, J. A. Hartley, M. J. Campbell, A. J. C. Leathem, and J. V. Woodside, "Effect of phytoestrogen and antioxidant supplementation on oxidative DNA damage assessed using the comet assay," Mutation Research, vol. 485, no. 2, pp. 169-176, 2001.

[27] R. C. M. Siow, F. Y. L. Li, D. J. Rowlands, P. de Winter, and G. E. Mann, "Cardiovascular targets for estrogens and phytoestrogens: Transcriptional regulation of nitric oxide synthase and antioxidant defense genes," Free Radical Biology and Medicine, vol. 42, no. 7, pp. 909-925, 2007.
[28] F. de Nigris, S. Williams-Ignarro, V. Sica et al., "Effects of a Pomegranate Fruit Extract rich in punicalagin on oxidationsensitive genes and eNOS activity at sites of perturbed shear stress and atherogenesis," Cardiovascular Research, vol. 73, no. 2, pp. 414-423, 2007.

[29] M. I. Gil, F. A. Tomas-Barberan, B. Hess-Pierce, D. M. Holcroft, and A. A. Kader, "Antioxidant activity of pomegranate juice and its relationship with phenolic composition and processing," Journal of Agricultural and Food Chemistry, vol. 48, no. 10, pp. 4581-4589, 2000.

[30] S. Z. Xu, W. Zhong, M. Ghavideldarestani, R. Saurabh, S. W. Lindow, and S. L. Atkin, "Multiple mechanisms of soy isoflavones against oxidative stress-induced endothelium injury," Free Radical Biology and Medicine, vol. 47, no. 2, pp. 167-175, 2009.

[31] C. Hermann, A. M. Zeiher, and S. Dimmeler, "Shear stress inhibits $\mathrm{H}_{2} \mathrm{O}_{2}$-induced apoptosis of human endothelial cells by modulation of the glutathione redox cycle and nitric oxide synthase," Arteriosclerosis, Thrombosis, and Vascular Biology, vol. 17, no. 12, pp. 3588-3592, 1997.

[32] I. Spyridopoulos, E. Brogi, M. Kearney et al., "Vascular endothelial growth factor inhibits endothelial cell apoptosis induced by tumor necrosis factor- $\alpha$ : Balance between growth and death signals," Journal of Molecular and Cellular Cardiology, vol. 29, no. 5, pp. 1321-1330, 1997.

[33] M. Artwohl, W. F. Graier, M. Roden et al., "Diabetic LDL triggers apoptosis in vascular endothelial cells," Diabetes, vol. 52, no. 5, pp. 1240-1247, 2003.

[34] M. Artwohl, K. Muth, K. Kosulin et al., "R-(+)- $\alpha$-lipoic acid inhibits endothelial cell apoptosis and proliferation: involvement of Akt and retinoblastoma protein/E2F-1," American Journal of Physiology, vol. 293, no. 3, pp. E681-E689, 2007.

[35] M. Artwohl, M. Roden, W. Waldhäusl, A. Freudenthaler, and S. M. Baumgartner-Parzer, "Free fatty acids trigger apoptosis and inhibit cell cycle progression in human vascular endothelial cells," The FASEB Journal, vol. 18, no. 1, pp. 146-148, 2004.

[36] M. Artwohl, T. Hölzebein, C. Fürnsinn et al., "Thiazolidinediones inhibit apoptosis and heat shock protein 60 expression in human vascular endothelial cells," Thrombosis and Haemostasis, vol. 93, no. 5, pp. 810-815, 2005.

[37] M. Artwohl, C. Fürnsinn, W. Waldhäusl et al., "Thiazolidinediones inhibit proliferation of microvascular and macrovascular cells by a PPAR $\gamma$-independent mechanism," Diabetologia, vol. 48, no. 3, pp. 586-594, 2005.

[38] M. Artwohl, A. Lindenmair, V. Sexl et al., "Different mechanisms of saturated versus polyunsaturated FFA-induced apoptosis in human endothelial cells," Journal of Lipid Research, vol. 49, no. 12, pp. 2627-2640, 2008.

[39] S. M. Baumgartner-Parzer, L. Wagner, M. Pettermann, J. Grillari, A. Gessl, and W. Waldhausl, "High-glucose-triggered apoptosis in cultured endothelial cells," Diabetes, vol. 44, no. 11, pp. 1323-1327, 1995.

[40] C. Ripoll, A. Coussaert, F. R. Waldenberger et al., "Evaluation of natural substances' protective effects against oxidative stress in a newly developed canine endothelial cell-based assay and in cell-free radical scavenging assays," Journal of Applied Research in Veterinary Medicine, vol. 10, no. 2, pp. 113-124, 2012.

[41] J. I. Ram and L. M. Hiebert, "Vitamin E protects porcine but not bovine cultured aortic endothelial cells from oxygenderived free radical injury due to hydrogen peroxide," Cell Biology and Toxicology, vol. 20, no. 1, pp. 55-67, 2004.

[42] R. M. McClain, E. Wolz, A. Davidovich, F. Pfannkuch, and J. Bausch, "Subchronic and chronic safety studies with genistein 
in dogs," Food and Chemical Toxicology, vol. 43, no. 10, pp. 1461-1482, 2005.

[43] N. P. Seeram, R. Lee, and D. Heber, "Bioavailability of ellagic acid in human plasma after consumption of ellagitannins from pomegranate (Punica granatum L.) juice," Clinica Chimica Acta, vol. 348, no. 1-2, pp. 63-68, 2004.

[44] T. R. Räthel, J. F. Leikert, A. M. Vollmar, and V. M. Dirsch, "The soy isoflavone genistein induces a late but sustained activation of the endothelial nitric oxide-synthase system in vitro," British Journal of Pharmacology, vol. 144, no. 3, pp. 394399, 2005.

[45] D. F. Horrobin and M. S. Manku, "Clinical biochemistry of essential fatty acids," in Omega-6 Essential Fatty Acids: Pathophysiology and Roles in Clinical Medicine, D. F. Horrobin, Ed., pp. 21-53, Wiley-Liss, New York, NY, USA, 1990.

[46] D. Kumar and B. I. Jugdutt, "Apoptosis and oxidants in the heart," Journal of Laboratory and Clinical Medicine, vol. 142, no. 5, pp. 288-297, 2003.

[47] H. Cai and D. G. Harrison, "Endothelial dysfunction in cardiovascular diseases: the role of oxidant stress," Circulation Research, vol. 87, no. 10, pp. 840-844, 2000.

[48] E. Sagols and N. Priyemenko, "Oxidative stress in dog with heart failure: the role of dietary fatty acids and antioxidants," Veterinary Medicine International, vol. 2011, Article ID 180206, 2011.

[49] P. Sestili, C. Martinelli, D. Ricci et al., "Cytoprotective effect of preparations from various parts of Punica granatum L. fruits in oxidatively injured mammalian cells in comparison with their antioxidant capacity in cell free systems," Pharmacological Research, vol. 56, no. 1, pp. 18-26, 2007.

[50] M. De Lorgeril, P. Salen, J. L. Martin, I. Monjaud, J. Delaye, and N. Mamelle, "Mediterranean diet, traditional risk factors, and the rate of cardiovascular complications after myocardial infarction: final report of the Lyon Diet Heart Study," Circulation, vol. 99, no. 6, pp. 779-785, 1999.

[51] G. Borges, W. Mullen, and A. Crozier, "Comparison of the polyphenolic composition and antioxidant activity of European commercial fruit juices," Food and Function, vol. 1, no. 1, pp. 73-83, 2010. 

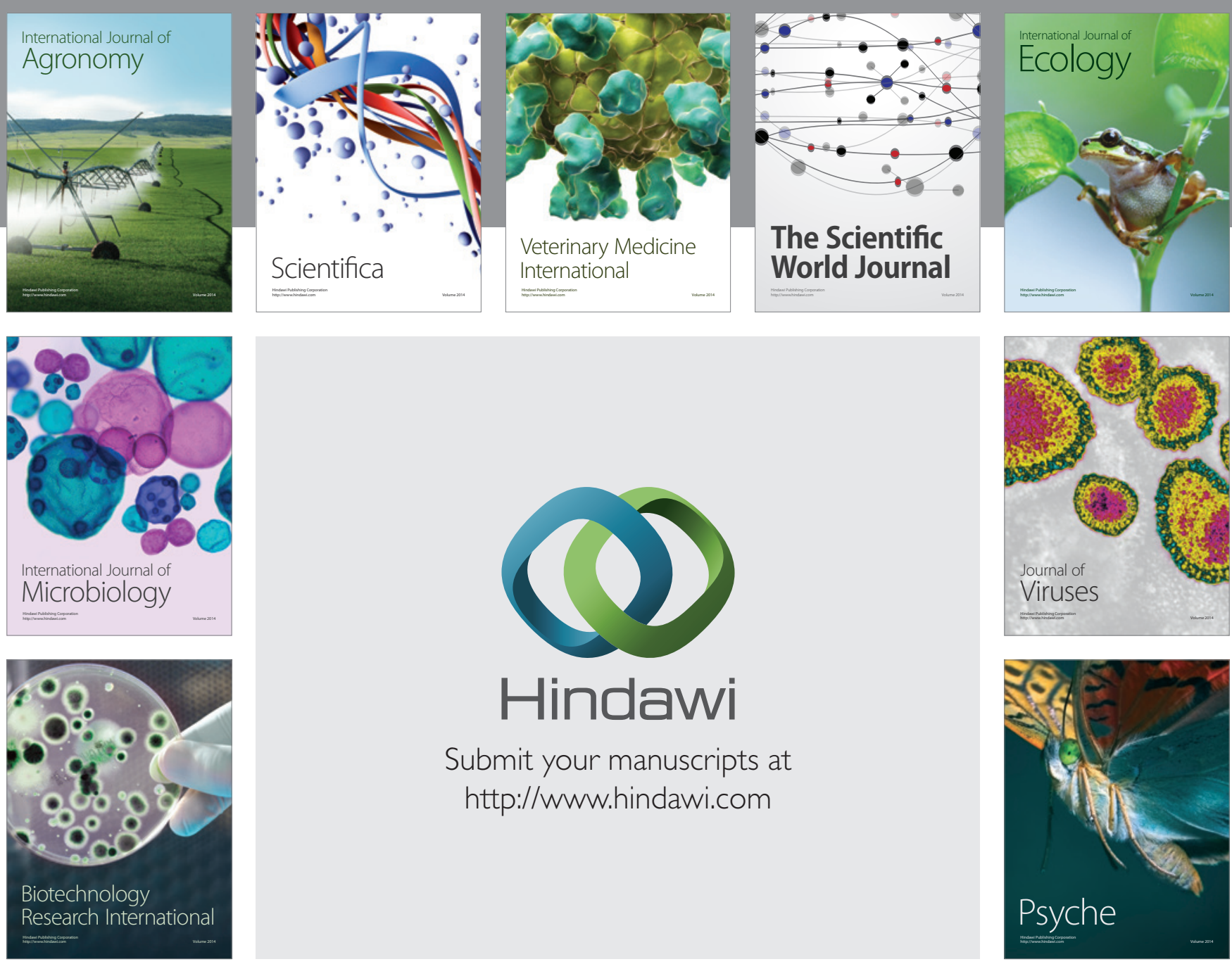

Submit your manuscripts at http://www.hindawi.com
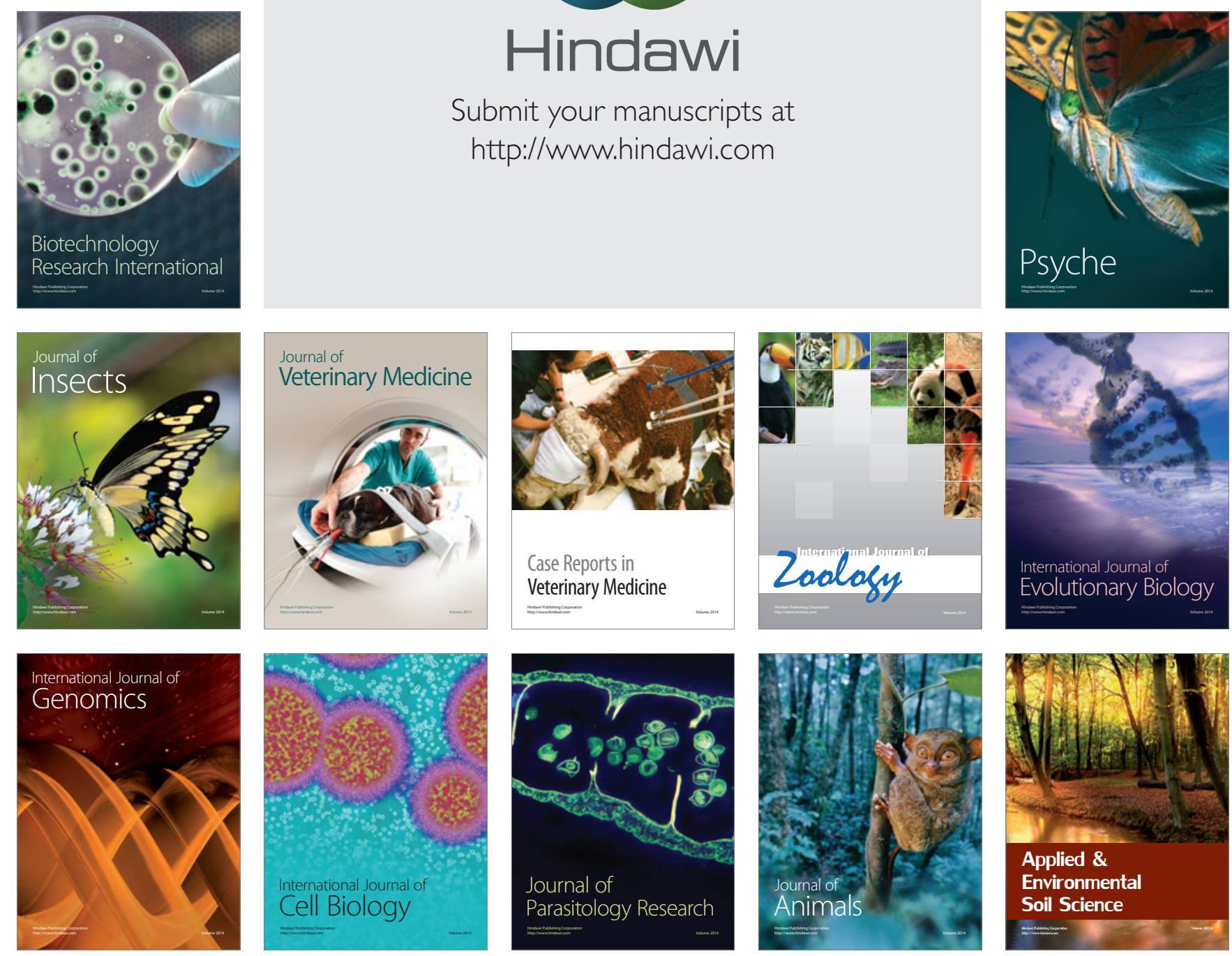